\title{
Lung Disease in Primary Antibody Deficiency
}

Verma $\mathrm{N}^{1}$, Grimbacher $\mathrm{B}^{1,2}$, Hurst $\mathrm{JR}^{3}$

1. Department of Immunology, Royal Free London NHS Foundation Trust, London, UK

2. Center for Chronic Immunodeficiency, Medical Center, University Hospital Freiburg, Germany

3. UCL Respiratory, University College London, London, UK

Corresponding Author:

j.hurst@ucl.ac.uk 


\section{ABSTRACT:}

This review summarises current knowledge on the pulmonary manifestations of primary antibody deficiency (PAD) syndromes in adults. We describe the major PAD syndromes, with a particular focus on Common Variable Immunodeficiency (CVID). Respiratory infection is a common presenting feature of PAD syndromes, and respiratory complications are frequent and responsible for much of the morbidity and mortality. Respiratory complications include acute infections, the sequelae of infection such as bronchiectasis, non-infectious immune-mediated manifestations - notably the development of granulomatous-interstitial lung disease (GLILD) in CVID - and an increased risk of lymphoma. Although minor abnormalities are detectable in the lung on CT scanning in the majority of patients with CVID, not all go on to develop lung complications. Mechanisms relating to the maintenance of lung health versus lung disease, and the development of bronchiectasis versus immune-mediated complications are now being dissected. We review current investigation, treatment and management strategies, and include key research questions, relating to both infectious and non-infectious complications of PAD in the lung.

\section{KEY MESSAGES:}

1. There are a wide spectrum of primary antibody deficiency (PAD) syndromes, reflecting the complexity of antibody reflection.

2. Respiratory infection is a common presenting feature of PAD, and respiratory clinicians should be alert to this.

3. Respiratory complications are also important in PAD - the focus of this review. These drive morbidity and mortality.

4. Respiratory complications can be considered as acute infections, the consequences of infection (such as bronchiectasis), immune mediated (notably granulomatous-interstitial lung disease, GLILD) in Common Variable Immunodeficiency (CVID) and other, such as an increased risk of lymphoma.

5. The pathways determining the development of different lung complications are now being defined.

6. Acute infections require prompt treatment, and use of antibiotic prophylaxis is recommended in PAD.

7. The treatment of bronchiectasis in PAD is not different from the management of bronchiectasis from any other cause, with the exception of immunoglobulin replacement.

8. The definition, investigation, treatment and management of GLILD and other interstitial diseases in PAD remains poorly defined. 


\section{Introduction}

This review summarises current knowledge on the pulmonary manifestations of primary antibody deficiency (PAD) syndromes in adults, with a focus on Common Variable Immunodeficiency (CVID), which is the most clinically significant PAD adult physicians are likely to encounter, and also the most well described. We will not consider PAD in children, upper airway (sino-nasal) manifestations of adult PAD, or lung disease in acquired antibody deficiency.

\section{Search Strategy and Selection Criteria}

A comprehensive literature review was conducted with support from the Clinical Effectiveness Enquiry Service at Royal Free London NHS Foundation Trust. MEDLINE and EMBASE were searched using text-words and subject headings relating to primary immune (antibody) deficiency syndromes, and lung diseases. The search strategy is provided in the supplementary appendix. The search was restricted to English language publications, and after removal of duplicates 2360 articles remained. The titles and where necessary abstracts were screened by one author (JRH) for suitability for inclusion. Additional citations were generated by examining the reference lists from the selected papers.

\section{The Spectrum of PAD Syndromes}

The production of specific antibodies targeted against pathogens is a key task for the mammalian immune system, and lack of a sufficient provision is the commonest immune defect seen in humans. A selective defect in IgA production is seen in 1 in 600 healthy blood donors ${ }^{[1]}$ and a lack of IgG production has a prevalence of about 1 in 25000 individuals $^{[2]}$.

There are various mechanisms which may result in PAD, summarised below and illustrated in Figure 1. Specific antibodies (immunoglobulins) are produced by memory B-cells and plasma cells (which derive from B-cells), thus the lack of B-cells, as seen in patients with in-born errors of B-cell development (e.g. following mutations in the X chromosomal Bruton's tyrosin kinase, Btk), will lead to agammaglobulinaemia. Other patients (with B-cells) may be able to produce IgM as a first line response to antigenic challenge, but are not then able to isotype switch to IgG or IgA because of genetic defects in the class switch recombination machinery. These patients are classified as "hyper IgM-", or having a class switch recombination defect. Others patients with B-cells are not capable of producing sufficient immunoglobulins to sustainably reach sufficient serum levels. This large group of patients are classified as Common Variable Immunodeficiency (CVID): "common" as this defect is one of the commoner immune defects in humans and patients have relatively 'similar' (common) defects in baseline laboratory investigations, and "variable" as the clinical presentation is heterogeneous (which is discussed further below). A further group of patients are not capable of producing a sufficient amount of one of the IgG subclasses. In humans, deficiency of IgG1 (mostly directed against protein antigens) and IgG2 (directed against polysaccharide antigens) are clinically 
relevant. Finally, there are patients who are able to produce immunoglobulins, but these are not specific enough to eliminate (neutralise) the targeted pathogen. These are called specific antibody deficiencies (SPAD). This failure most frequently concerns the polysaccharides present in the capsule of encapsulated bacteria such as Pneumococcus and Haemophilus influenzae, highly relevant pathogens in respiratory diseases. Antibodies are needed to opsonise capsulated bacteria thus activating the complement system. This explains why in antibody-deficient patients respiratory infections are prevalent, and pose a clinical challenge. Further details about these sub-groups are provided in the sections below.

FIGURE 1: A summary of B-cell development, with reference to PAD disease pathogenesis.

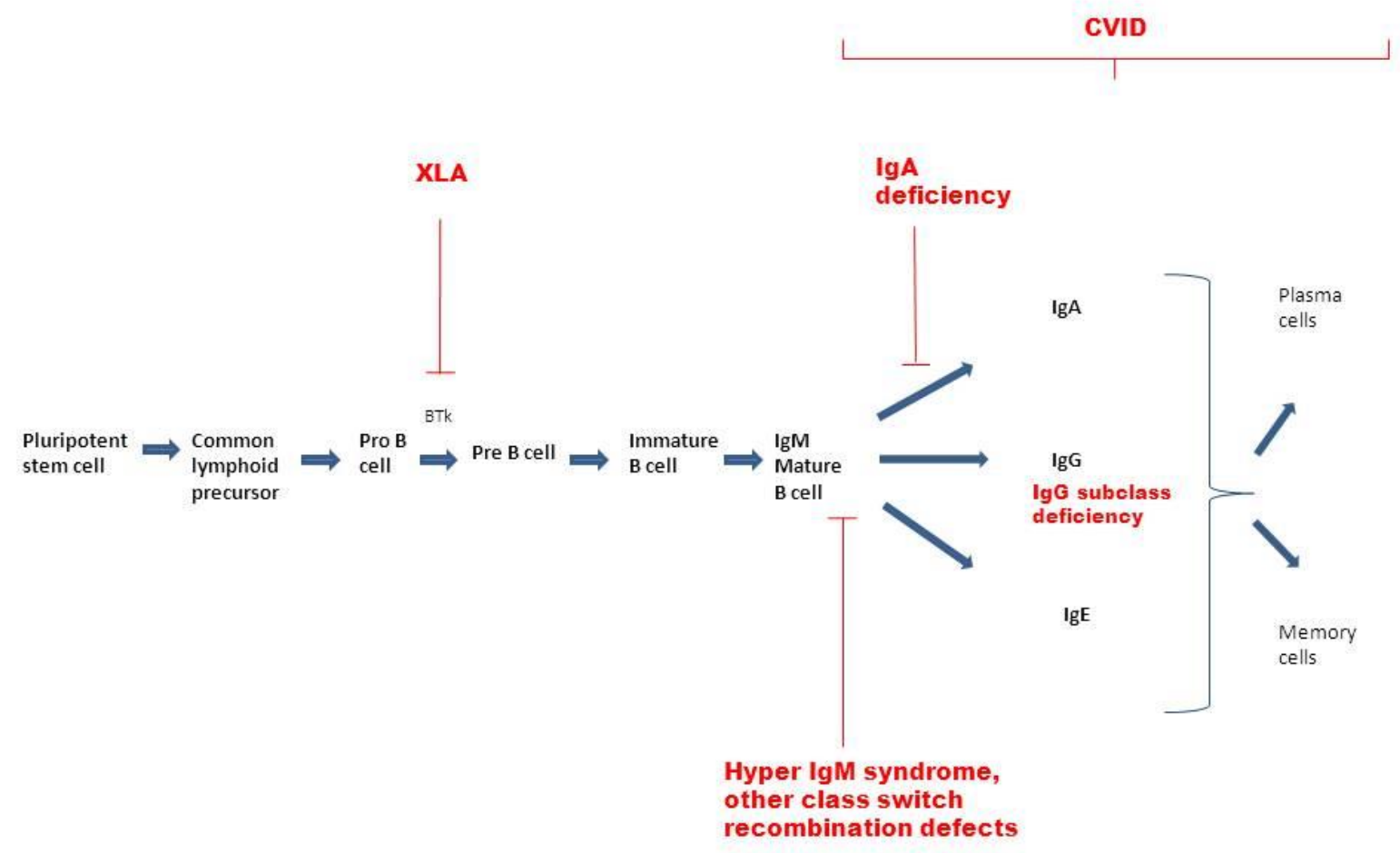

\section{HYPER IgM SYNDROMES}

Class switch recombination (CSR) syndromes (hyper-IgM syndromes) can be caused either by an absence of sufficient B-cell co-stimulation such as the lack of CD40:CD40-ligand interaction, or by a defect in the CSR machinery, for example due to mutations in AID (Activation-induced cytidine deaminase, UNG (uracil DNA glycosylase), or INO80 and others. CSR syndromes are characterised by normal or elevated levels of IgM but low IgG and IgA, and therefore susceptibility to respiratory infection ${ }^{[3]}$. 


\section{COMMON VARIABLE IMMUNODEFICIENCY DISORDERS (CVID)}

CVID, the most clinically significant PAD, forms part of a heterogeneous group of antibody disorders. Its prevalence is estimated to be 1:25-50000, affecting males and females equally. Recurrent bacterial infections, commonly of a sino-pulmonary origin are the hallmark feature of this condition. Known complications include cytopaenias, autoimmunity, granuloma-formation affecting numerous organs (most commonly the lungs as discussed further below, and liver), splenomegaly, gastrointestinal tract involvement and lymphoproliferative disorders. Multi-organ involvement is often seen, with end organ damage. Though primarily a B-cell disorder, T-cell abnormalities may also occur in CVID.

Symptoms of CVID can occur at any age, though tend to peak in early childhood, late adolescence and in the third and fourth decades. The high variability in clinical features seen in CVID may result in a delay in diagnosis. It has been reported that the delay from the onset of initial symptoms to formal diagnosis averages around five years in western countries ${ }^{[2]}$ with a delay in initiating appropriate management.

A low IgG level with a low IgA and/or IgM level combined with a poor vaccination response to a polysaccharide based vaccination should always raise suspicion of a diagnosis of CVID. The clinical criteria for a probable diagnosis of CVID as per the Registry Working Party of the European Society of Immunodeficiency ${ }^{[4]}$ (ESID) are listed in Table 1.

TABLE 1: ESID registry criteria for a probable diagnosis of CVID [4].

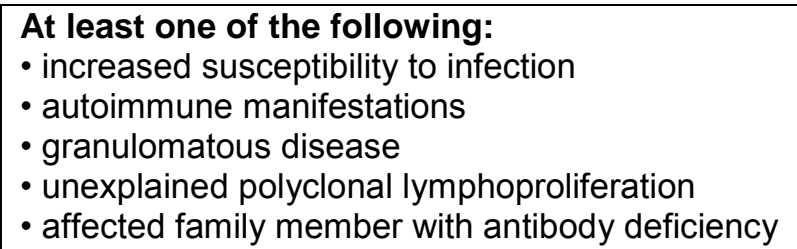

AND marked decrease of $\lg G$ and marked decrease of $\lg A$, with or without low $\lg M$ levels (measured at least twice; $<2 S D$ of the normal levels for their age);

AND at least one of the following:

- poor antibody response to vaccines (and/or absent isohaemagglutinins); i.e. absence of protective levels despite vaccination where defined

- low switched memory B cells ( $<70 \%$ of age-related normal value)

AND secondary causes of hypogammaglobulinaemia have been excluded

AND diagnosis is established after the 4th year of life (but symptoms may be present before)

AND no evidence of profound T-cell deficiency

X-LINKED AGGAMAGLOBULINAEMIA (XLA):

As an X-linked disorder, XLA occurs almost exclusively in males. A defect in the maturation of antibody-producing B-cells results in absent levels across all classes of immunoglobulins. Most patients are diagnosed pre-school, once protective maternal antibodies have waned. A later diagnosis has been reported in those with a negative family history, at an average age of 5.4 years compared to 2.6 years in those with a positive family history ${ }^{[5]}$. Infection with encapsulated organisms such as Streptococcus pneumoniae and Haemophilus influenzae are common. Sino- 
pulmonary infections, most often otitis, are the most frequent presentation. Other infections that may occur include conjunctivitis, septic arthritis, osteomyelitis, and enteroviral infection manifesting as diarrhoea and encephalitis. Agammaglobulinaemia may also be seen as an autosomal recessive condition.

\section{SELECTIVE IgA DEFICIENCY}

Dimeric IgA2 is the most abundant antibody in mucosal secretions and selective IgA deficiency (slgAD) is the most common primary immunodeficiency. Although the prevalence of slgAD is 1 in 600 in Caucasians ${ }^{[1]}$, less than $10 \%$ of subjects have clinical symptoms, suggesting the presence of compensatory mechanisms at the mucosal surface. There is evidence that patients with slgAD are at increased risk of both upper and lower respiratory tract, and gastro-intestinal infections ${ }^{[6]}$.

\section{IMMUNOGLOBULIN SUB-CLASS DEFICIENCY}

IgG is the predominant antibody in the circulation, and composed of four sub-classes each with structural differences that confer functional variation. IgG subclass deficiency may occur in isolation or in combination with deficiency of other IgG subclass or immunoglobulin isotypes. IgG2 deficiency predominates in childhood. IgG4 is generally present in very low concentrations and absent in about $8 \%$ of Caucasians. Hence deficiency in this alone is not clinically significant. IgG1 and IgG2 subclass deficiencies present in a similar manner to other PADs with recurrent upper and lower respiratory tract infections, sinusitis and otitis media, and an increased vulnerability to invasive infection with encapsulated organisms. The nature of the pathogen susceptibility may indicate the subclass deficiency: for instance, those particularly lacking IgG2-polysaccaride-specific antibodies lack an important protective mechanism against encapsulated organisms such as Streptococcus pneumonia and encapsulated forms of Haemophilus influenzae.

\section{SPECIFIC ANTIBIODY DEFICIENCY}

The diagnosis of Specific Antibody Deficiency (SPAD) arises in those with normal total immunoglobulin levels but an impaired response to, for example, polysaccharide antigens, detected through the use of polysaccharide test vaccinations such as Pneumovax-23. An adequate vaccination response is usually one that is $4 x$ greater than the baseline antibody level. To exclude SPAD fully, we measure both the overall Pneumococcal titre as well as serotype specific pneumococcal antibody levels to 13 serotypes, both pre and post vaccination. Impaired responses are present in children below the age of two years, who still have an immature immune response to encapsulated bacteria. However, healthy children will grow out of this and only if they do not can SPAD be diagnosed. Other components of the immune system are usually intact and therefore adults present with recurrent upper and lower respiratory tract infections, but often no other organ involvement. 


\section{GOOD'S SYNDROME}

The presence of a thymoma with associated hypogammaglobulinaemia comprises Good's syndrome. Hypogammaglobulinaemia is seen in up to $10 \%$ of patients with a thymoma. The clinical picture and laboratory investigations are variable but patients essentially present in a similar way to other PAD with an increased burden of bacterial infections with encapsulated organisms, but also opportunistic viral and fungal infections. Urinary tract infections, skin infections, diarrhoea and autoimmunity have all been described. Associated pathogens include cytomegalovirus and herpes simplex, candida, and Pneumocystis carinii.

\section{RECENT MUTATIONS DESCRIBED IN PAD:}

Over the past few years, a number of specific mutations have been identified in a proportion of patients with PAD, some of which may predispose to specific lung manifestations.

APDS. The recently described Activated PI3K- $\delta$ syndrome (APDS) ${ }^{[7-11]}$ is a monogenetic autosomal dominant defect that leads to the activation of lymphocytes resulting in uncontrolled lymphoproliferation. This is associated with impaired vaccine responses, reduced serum IgG2 and recurrent respiratory infection with airway damage. In the lungs, the expanded lymphoid tissue, (including but not limited to the lymph nodes) compresses the bronchi, leading to characteristic radiologic and bronchoscopic appearances. These lymphoid aggregates may result in post-stenotic pneumonia. We will not further consider this entity.

CTLA-4 deficiency. CTLA-4 deficiency is a recently described autosomal dominant immune dysregulation syndrome characterised by an activated T-cell compartment ${ }^{[12,13]}$. As regulatory T-cells in this condition fail to exert their immune-regulatory effect, auto-aggressive immune cell infiltrations attack the lungs and often lead to granulomatous lymphocytic interstitial lung disease, which is described further below.

Early recognition and diagnosis of PAD is vital. We suggest that all patients with underlying structural lung disease should have baseline immunoglobulin levels measured at least once, including IgG, IgA and IgM, to exclude PAD. If clinical suspicion for PAD remains, referral to an Immunologist should be sought for more specialist investigations. Importantly, this is not reflected in national and international guidelines across the spectrum of lung disease.

\section{Lung Disease in PAD}

The respiratory manifestations of PAD follow two basic pathophysiological mechanisms, i) the sequelae of recurrent acute infections and ii) immune-mediated pathology. Hence, two distinct patterns of chronic lung disease develop: bronchiectasis and interstitial disease. These may be 
readily distinguished using computed tomography (CT), as illustrated in Figure 2. It is now apparent that the presence of these two conditions is associated with different immune profiles, at least when examined in retrospective studies. Whether such parameters predict the development of future lung complications at the time of diagnosis remains to be assessed, but would be a major clinical development. Interstitial disease, for example, has been associated with altered CD4:CD8 T-cell proportionality (ratio increased), elevated IgM, a history of autoimmune haemolytic anaemia or immune thrombocytopenic purpura and tends to occur at a younger age. In contrast, bronchiectasis tended to affect an older age group, and is associated with a history of pneumonia and a CD4+ T-cell count lower than 700 cells $/ \mu]^{[14]}$. This is summarised in Figure 3.

FIGURE 2: Typical lung-window CT findings in bronchiectasis (left, A) and interstitial lung disease (right, B), both in patients with underlying antibody deficiency. Bronchiectasis is seen as bronchial wall thickening, and dilatation of the bronchus in relation to the accompanying vessel. The interstitial scan shows multiple peri-bronchial nodules and ground-glass change. Fibrosis is seen in patients with more advanced interstitial disease.

A

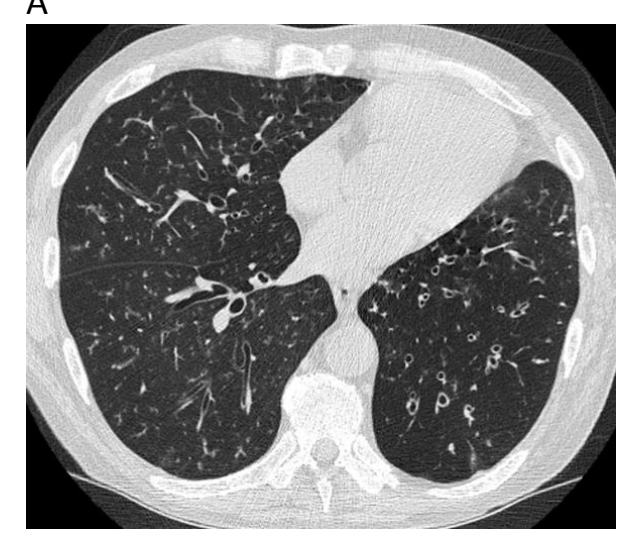

B

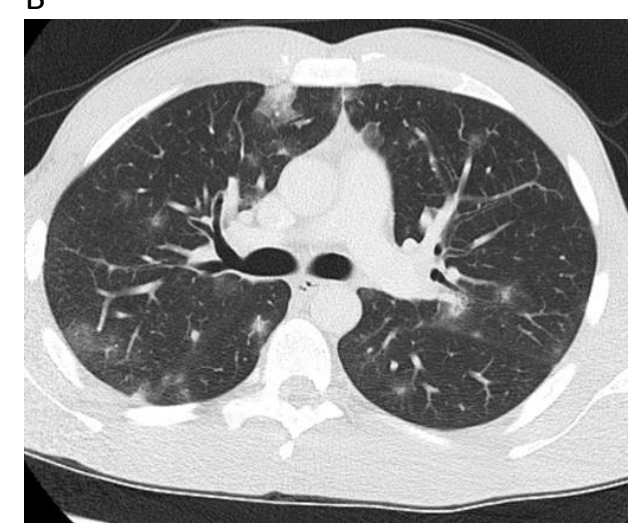

FIGURE 3: Hypothetical scheme outlining pathways to the development of lung disease in PAD. Ig= immunoglobulin; $G L I L D=$ granulomatous interstitial lung disease; $M B L=$ mannose binding lectin. 


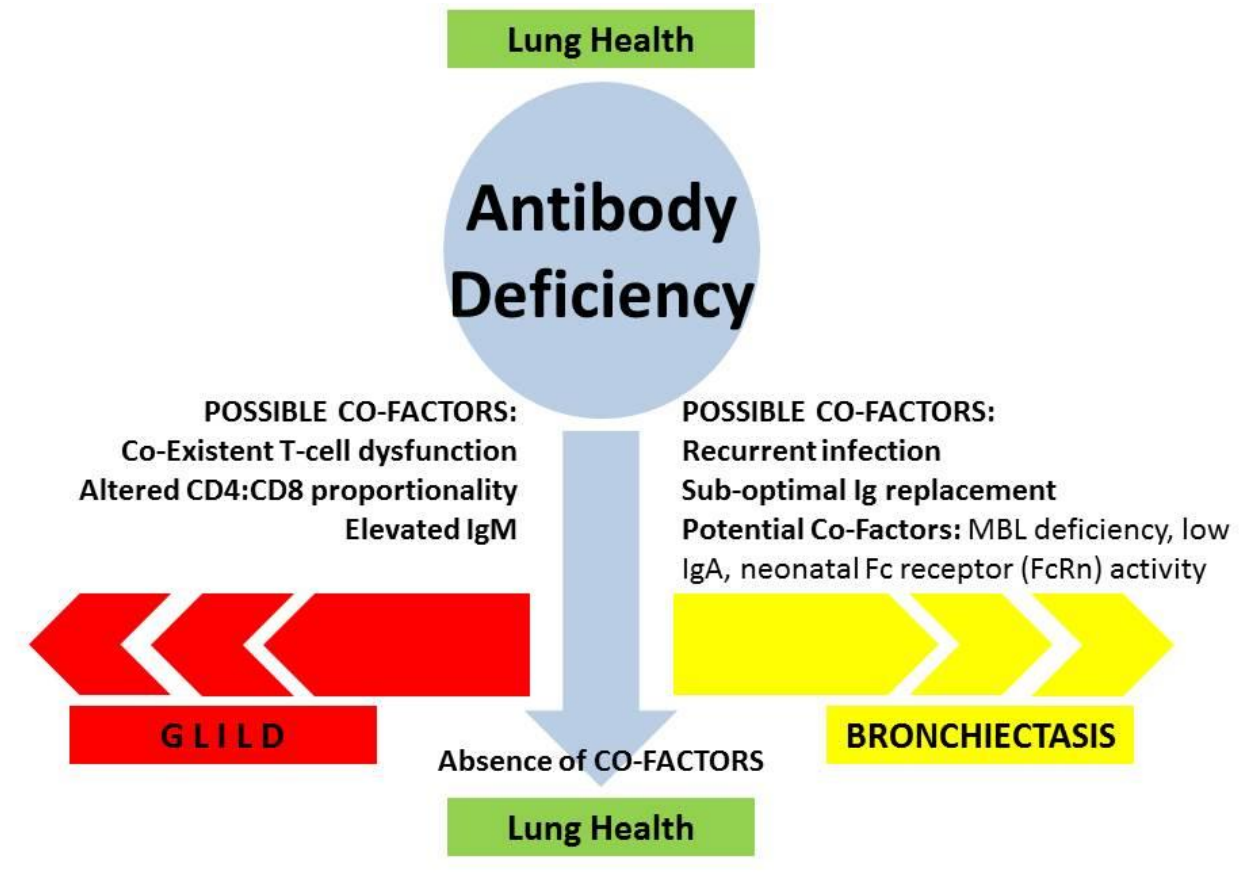

The detection of lung involvement in PAD is important because of effects on quality of life ${ }^{[15,16]}$ and mortality ${ }^{[17]}$. In a seminal study, mortality in CVID has been linked to both structural and functional lung impairment ${ }^{[17]}$. A diagnosis of structural lung disease included interstitial changes on CT, granulomatous or lymphocytic infiltrates confirmed on lung biopsy, bronchiectasis, or a combination of these. Functional lung disease included restrictive or obstructive lung pathology, reduced diffusion capacity, and/or reduced oxygen saturation.

Classically bronchiectasis, at least when severe, is associated with airflow obstruction (decreased $\mathrm{FEV}_{1} / \mathrm{VC}$ ratio) whilst interstitial change is typically associated with restrictive abnormalities on pulmonary function, including impairment in gas transfer (decrease in $\mathrm{DL}_{\mathrm{co}}$ ) ${ }^{[18]}$. When spirometry is normal, early abnormality may be detectable by changes in mid-expiratory flows.

The prevalence of lung disease is lower in XLA than CVID, likely reflecting both earlier diagnosis and the additional immune dysregulation seen in $\mathrm{CVID}^{[19]}$.

It is known that over $90 \%$ of patients with CVID have abnormalities present on CT scan of the lungs ${ }^{[20]}$, typically mild and present even in patients who are asymptomatic ${ }^{[18]}$. We recommend a baseline CT chest at diagnosis of PAD to identify respiratory complications early in the illness, allowing prompt treatment and providing a baseline from which to assess future change. In those who are asymptomatic, and in the absence of other biological markers, this results in the clinical problem of identifying which patients will go on to develop progressive lung disease, mandating repeat CT assessments (in our practice five yearly to limit lifetime radiation exposure with repeated scanning, and with annual lung function screening). Whilst certain cell populations (e.g. memory Bcells) may be absent or reduced in those with chronic lung disease ${ }^{[21]}$, it is not known if the association with chronic lung disease is cause or effect. In those with symptoms, the commonest problems are recurrent infections, at a median frequency of 2.5 events per year, and productive cough $^{[22]}$. 
Current assessment of patients with lung involvement requires serial lung function testing, six monthly to yearly depending on severity, and repeat imaging, as lung involvement can progress despite immunoglobulin replacement ${ }^{[23]}$. Repeated exposure to ionising radiation is a concern, particularly in patients whose genetic defect affects DNA recombination and DNA repair, leading to radio-sensitivity. Protocols should therefore minimise the radiation exposure. MRI has been explored as a tool to replace $\mathrm{CT}^{[24]}$, but scan resolution remains a challenge as illustrated in Figure 4 . However, MR sequences may give additional functional information to that provided by $\mathrm{CT}$, for example regarding the cellularity of pulmonary nodules. 
FIGURE 4: $A$ comparison of MR ( $A$, left) and CT images ( $B$, right) from a patient with CVID and interstitial lung disease. Note that the MR provides less special resolution, but on these T2weighted images the presence of oedema (high signal) within some nodules becomes apparent. CT shows bilateral ground-glass and more dense nodular infiltration, only some of which is visible on MRI.

A

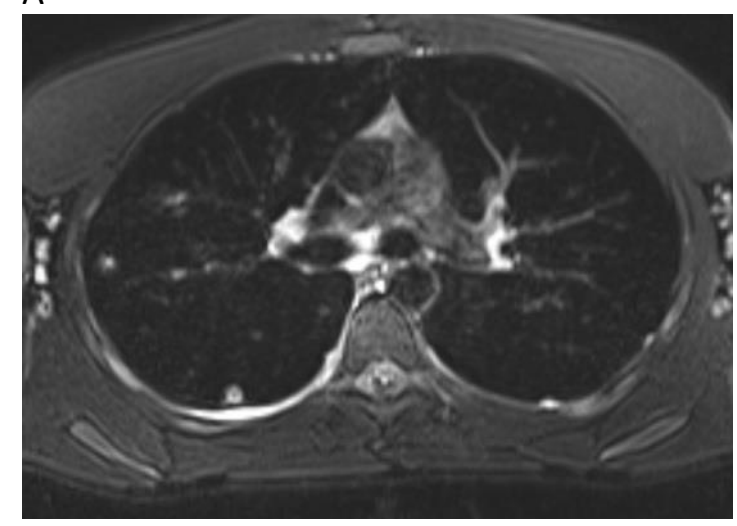

B

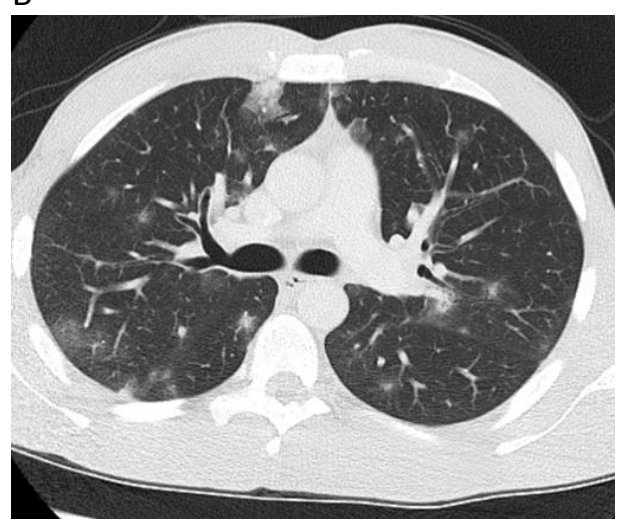

Little is known about rates of lung function decline in PAD, or the factors affecting this. In a small study of 37 patients over an average period of 7.6 years ${ }^{[25]}$, the average decline in $\mathrm{FEV}_{1}$ was $45 \mathrm{ml} /$ year, greater than would be expected in a normal population (typically $30 \mathrm{ml} / \mathrm{year}$ ), and similar to that expected in a susceptible smoker. Decline was more rapid in patients diagnosed at a younger age and was more rapid in XLA than CVID patients (65 vs. $36 \mathrm{ml} /$ year). Decline was reduced in patients on higher doses of immunoglobulin replacement (irrespective of trough level). The relationship with antibiotic use and infection frequency remains to be defined ${ }^{[26]}$. The concept that immunoglobulin replacement can be used to reduce lung function decline requires further confirmation.

It is important to draw a distinction between disease severity which can be assessed using crosssectional measures such as a single lung function test or $\mathrm{CT}$, and the disease activity, or speed of progression of lung disease. We reported in a small study in patients with PAD that the rate of FEV 1 decline (but not absolute $\mathrm{FEV}_{1}$ or extent of disease on $\mathrm{CT}$ ) was directly proportional to the systemic acute phase response (and that systemic inflammation was related to that in the airway) ${ }^{[16]}$.

\section{Acute Infections}

Acute infections contribute significantly to the costs of care in patients with PAD ${ }^{[27]}$. It is clear that immunoglobulin replacement is effective in reducing the risk of infection, including pneumonia, otitis media, and systemic infections in patients with XLA, hyper-IgM and CVID ${ }^{[28-30]}$. Despite immunoglobulin replacement, susceptibility to infection remains variable ${ }^{[31]}$ and may relate to low IgA concentration. Trough levels relate to infection susceptibility in meta-analysis ${ }^{[30]}$. Over a five year period in 201 patients, $21 \%$ of CVID patients and $24 \%$ of XLA patients were infection free. A 
higher steady-state IgG in subcutaneous replacement is also associated with fewer acute infections ${ }^{[32]}$. Both Intravenous and subcutaneous immunoglobulin replacement have been shown to be equally effective routes of administration in reducing infection burden. There are no specific guidelines for an 'adequate' trough level though generally the aim is to be above the lower limit of the normal IgG range. It has been proposed that rather than a particular trough level, the number of breakthrough infections should determine the immunoglobulin dose for each individual patient ${ }^{[29]}$.

With regard to the causative pathogens, bacterial infections are most important, typically with Streptococcus, Haemophilus and Staphylococcus, at least when employing culture-based detection. Viral infections, including rhinovirus infection, may be both more frequent and more prolonged in $\mathrm{PAD}^{[33]}$. Pathogens that are considered 'opportunistic' in the context of conditions such as HIV, for example Pneumocystis and CMV, do not appear common in the context of PAD lung disease ${ }^{[34}$. This likely reflects the relative preservation of adequate T-cell function in many patients with PAD.

Acute infection should be managed as for guidance in patients without underlying PAD, but treatment may need to be prolonged for 10 to 14 days in patients with established bronchiectasis. Evidence is scarce on appropriate antibiotic prophylaxis and its use, in PAD. In our clinical experience, similar to other centres nationwide, antibiotic prophylaxis has been associated with a substantially reduced infection burden. Our practice is to offer antibiotic prophylaxis to all patients with PAD and recurrent infections, especially when there are known structural lung changes. Preventing infections is of key importance in reducing long term morbidity. We do not routinely use antifungal prophylaxis, in the absence of data that fungal pathogens are a common cause of infection in our cohort of CVID patients. In those with evidence of significantly reduced T cell counts with or without T cell dysfunction, we prefer co-trimaxazole as antibiotic prophylaxis to cover both bacterial and fungal organisms.

\section{Bronchiectasis in PAD}

Clinically significant bronchiectasis is defined as permanent abnormal dilatation of the airways in conjunction with persistent or recurrent bronchial sepsis ${ }^{[35]}$. The clinical picture is therefore one of chronic productive cough, susceptibility to recurrent exacerbations, and breathlessness in those with more extensive disease.

Despite the importance of detecting primary immunodeficiency as a (treatable) cause of bronchiectasis, a UK national audit reported that only $68 \%$ of patients with bronchiectasis had documented serum immunoglobulin assay ${ }^{[36]}$.

The prevalence of bronchiectasis in CVID, 23\% across a European cohort of 902 subjects, varies considerably by centre ${ }^{[37]}$. In part this reflects the difficulty in differentiating minor degrees of bronchiectasis from healthy lung, but even in the absence of bronchiectasis there may be airway wall thickening, with secondary atelectasis and gas-trapping visible on $\mathrm{CT}^{[38]}$. 
We have previously reported that health-status in patients with PAD and bronchiectasis is largely driven by the respiratory involvement, and related to the degree of airflow obstruction, breathlessness, and respiratory infection frequency ${ }^{[16]}$. Thus, preventing infections might be expected to maintain or improve health-status, and there is now evidence for strategies such as macrolide antibiotics to achieve this in patients with bronchiectasis (though some trials excluded PAD populations) ${ }^{[39-41]}$.

As not all patients with PAD develop bronchiectasis, it is clear that co-factors must be important and it has been suggested that additional deficiency of mannose-binding lectin (MBL), a protein component of the complement pathway, may be one such variable ${ }^{[42]}$. Low IgA has also been associated with the development of bronchiectasis ${ }^{[31]}$, as has activity of the neonatal Fc receptor (FcRn) activity ${ }^{[43]}$.

The management of bronchiectasis in PAD is not different from the management of bronchiectasis from any other cause ${ }^{[39]}$, with correction of the underlying defect (in this case optimal immunoglobulin replacement), sputum clearance training from a respiratory physiotherapist and prevention of further infection which usually involves prophylactic antibiotics in patients with PAD. There is a paucity of data about antibiotic choice, with the greatest evidence for macrolides ${ }^{[39-41]}$ though with the caveat that evidence specifically in PAD is poor ${ }^{[44]}$. Whether the beneficial effect of macrolides is due to a classical antibiotic effect remains to be clarified and anti-inflammatory action may also be relevant. There is a general consensus ${ }^{[35]}$ to avoid ciprofloxacin prophylaxis given the utility of this drug in treating Pseudomonas. Patients with PAD may not make appropriate vaccine responses to the influenza and Pneumococcal vaccines that are recommended as part of usual bronchiectasis care.

There remains something of a paradox in the concept that IgA provides most mucosal defence, yet IgA deficiency tends to result in less severe lung disease than that associated with IgG deficiency, and replacement of IgG into the systemic circulation is able to reduce the frequency of respiratory infections ${ }^{[17]}$. This may be because whilst IgA is mostly present in the upper airways, IgG is the most prevalent immunoglobulin in the alveolar space.

Whilst primarily prescribed to reduce the incidence of acute infection, there is data from a small study in thirteen patients ${ }^{[45]}$ that immunoglobulin replacement may be associated with beneficial effects on airway inflammation, including a reduction in inflammatory cell count, and increased mucus transportability.

Studies using culture and PCR have demonstrated the presence of potentially pathogenic bacteria and viruses from the lungs of patients with PAD even when clinically stable $\mathrm{e}^{[47]}$. Haemophilus influenza is the commonest pathogen identified by culture ${ }^{[22]}$. However, our understanding of the lung microbiome has been transformed over recent years, such that we now understand that the differences between health and disease, and stable and exacerbated states are associated with differences in bacterial populations ${ }^{[46]}$. While research is underway, there are no published data exploring how the respiratory microbiome may be altered in patients with underlying PAD or, indeed, whether changes in the microbiome may predispose to rather than result from specific types of lung disease. 


\section{Interstitial Lung Disease in PAD}

Any interstitial lung disease may be seen in association with PAD, but the most common, the most heavily researched, and the one most closely associated with poor clinical outcomes is currently considered clinically as an entity called granulomatous-interstitial lung disease (GLILD). This term, helpful to clinicians, is less widely accepted by pathologists and requires the development of a precise clinico-pathologico-radiological definition comparable to other ILD. Radiologically, the typical pattern of interstitial lung disease in PAD is a generalised diffuse reticular change, often with ground-glass appearance, and a lower lobe predominance. In one report, large, ill-defined bronchocentric nodules or small randomly distributed nodules were found in $50 \%$, invariably in association with reticular change and $38 \%$ had thoracic lymphadenopathy ${ }^{[48]}$. This is illustrated in Figure 2.

Histologically, this condition is characterised by both granulomatous, and lymphoproliferative features including non-necrotising granuloma, lymphocytic interstitial pneumonitis and follicular bronchiolitis (Figure 5). Recently, the lymphoid hyperplasia has been carefully characterised and found to contain a variable preponderance of B- and T-cells (more commonly T-cell predominant), organised into distinct zones, with evidence of active cellular proliferation ${ }^{[49]}$.

\section{FIGURE 5: Histology of GLILD demonstrating a polymorphous infiltrate of lymphocytes, plasma} cells and macrophages.

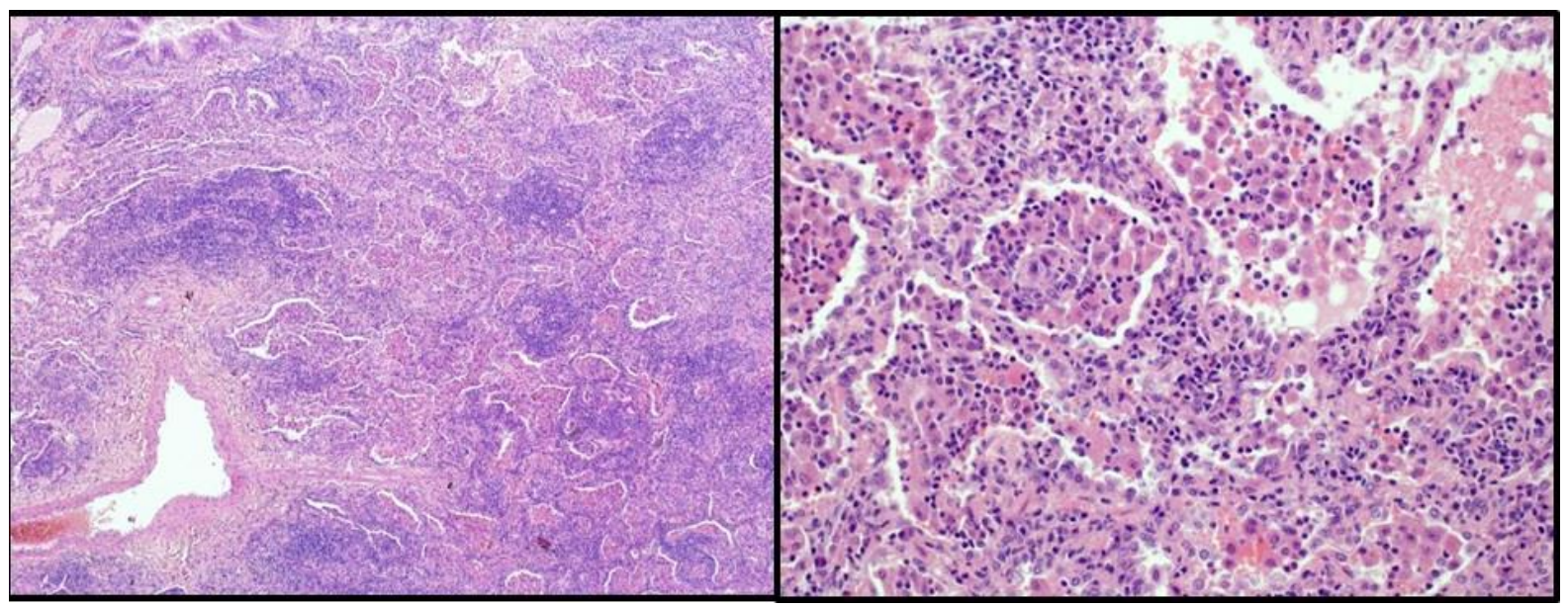

GLILD is perhaps best considered as the pulmonary manifestation of a systemic syndrome associated with autoimmune cytopaenia, splenomegaly, enteritis and adenopathy. GLILD is not seen in XLA, suggesting that T-cell dysfunction is central to the pathogenesis, though the detailed mechanisms remain poorly understood. Various studies have examined the role of viruses such as herpes viruses in driving lymphocyte proliferation without consistent results. 
Clinically, patients experience cough and breathlessness. Impairment in gas transfer appears to be the most frequent abnormality on lung function testing ${ }^{[50]}$. As the natural history of GLILD is not well documented in PAD patients, observational studies such as STILPAD ${ }^{[51]}$ are on-going to further examine this complication.

Our approach, summarised as Figure 6, is to confirm the diagnosis of GLILD at surgical (videoassisted thoracic surgery, VATS) lung biopsy. This is not a trivial procedure, but the relative preponderance of different cell populations may then be used to guide second-line therapy. Direct evidence in support of this approach remains limited ${ }^{[49]}$. The differential diagnosis includes infection, other ILD, and lymphoma. We perform broncho-alveolar lavage, prior to biopsy, to exclude infection. It has been suggested that GLILD may be associated with a BAL lymphocytosis $(>20 \%)$ in the majority of patients ${ }^{[52,53]}$, but whether further analysis of cells retrieved at BAL is able to avoid surgical biopsy has yet to be properly examined. The CD4/CD8 preponderance has varied between studies, and a higher ratio has been associated with a more favourable prognosis in terms of lung function decline in one study ${ }^{[53]}$, but not in another ${ }^{[54]}$. It is recognised that abnormalities present in blood lymphocyte populations in CVID may also be present in $B A L^{[55]}$, but in general there is a paucity of data on lung lymphocyte populations in CVID

FIGURE 6: An approach to the diagnosis and management of GLILD in CVID.

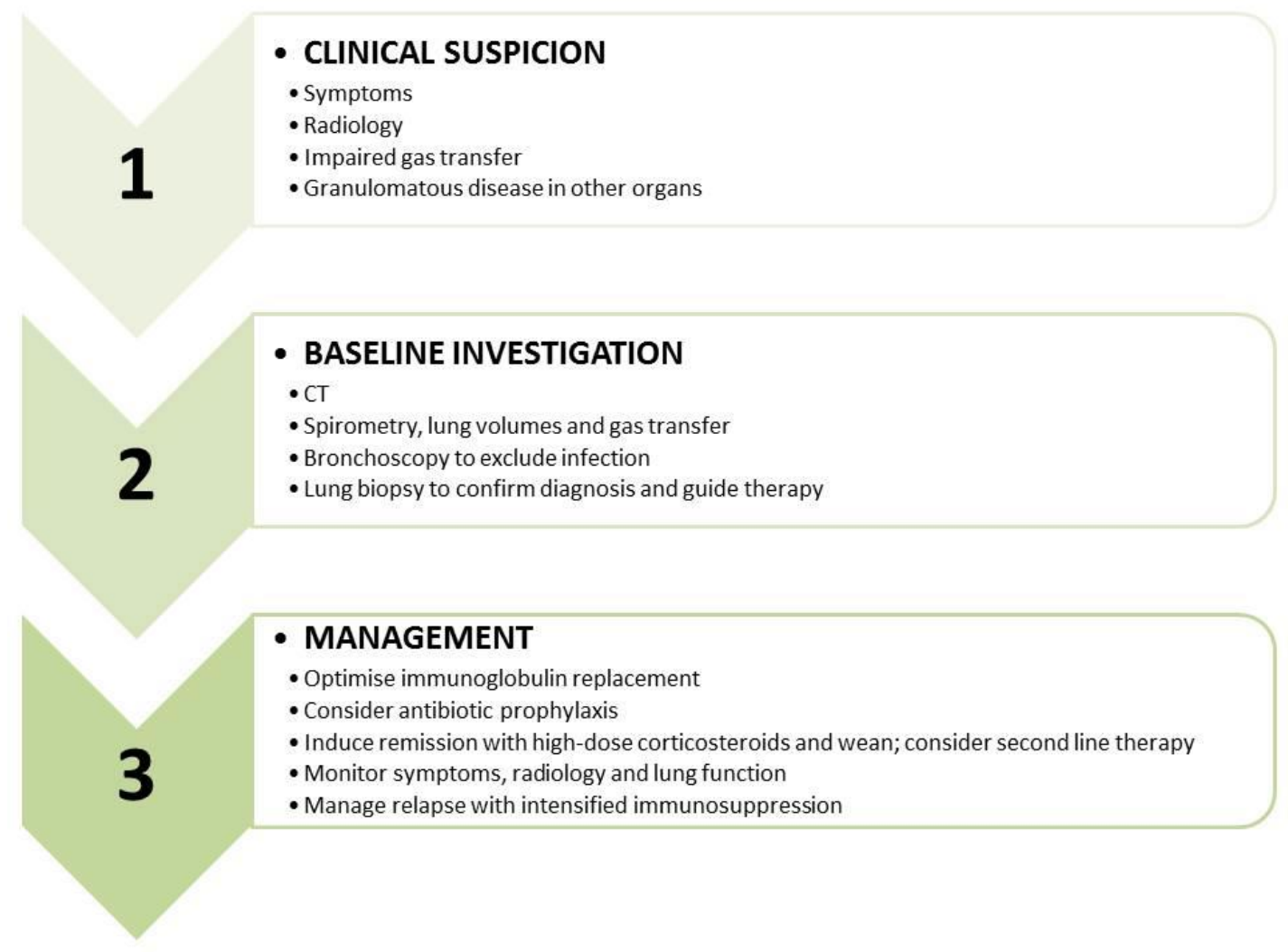

One study ${ }^{[53]}$ included an assessment with trans-bronchial biopsy but this was not adequate to make a firm diagnosis in over $2 / 3$ of patients. 
Disease progression can be seen in patients on immunoglobulin replacement ${ }^{[23]}$ There are no appropriate studies examining whether immunoglobulin optimisation alone can improve lung function in GLILD. High dose corticosteroids are often used as first-line therapy, although the evidence is weak ${ }^{[50,56]}$, and a complete response may require combination therapies since response to steroids may be absent in around half of patients ${ }^{[50]}$. Based on our clinical experience we start at $1 \mathrm{mg} / \mathrm{kg} /$ day until there is maximal improvement in lung function and radiology and then wean (Figure 6). When abnormalities are visible on plain radiography, this can be used to monitor therapy in preference to CT. Patients are monitored for CD4 lymphopenia secondary to corticosteroid immunosuppression and prophylaxis for Pneumocystis considered if the CD4 count falls below $200 \times 10^{9} /$. There is no specific evidence that this is beneficial in CVID patients but there is increasing evidence of this having a protective effect in non HIV immunocompromised patients ${ }^{[57]}$

Case reports suggest rituximab can improve pulmonary function ${ }^{[49]}$, and has been used in conjunction with azathioprine (targeting T-cells) resulting in resolution of spirometry and imaging abnormalities ${ }^{[58]}$ in a small series. Other T-cell agents that have been tried include ciclosporin ${ }^{[59]}$ and abatacept. Case reports exist for infliximab ${ }^{[60]}$. We have clinical experience using mycophenolate as second-line therapy.

Bone-Marrow Transplant has been performed in selected patients with GLILD ${ }^{[61]}$.

Although larger datasets are required, it is clear that GLILD is associated with reduced life expectancy in CVID ${ }^{[62]}$. There is little data on outcomes of lung transplantation, which has on occasion been successfully performed although reviews suggest outcomes may be variable ${ }^{[63]}$, and granulomas have been reported to recur ${ }^{[22]}$.

It is unusual for patients with underlying PAD and histologically confirmed GLILD to display no symptoms. In the rare instances where this is the case, treatment should still be considered to prevent further end organ damage.

\section{Other}

Organising pneumonia (previously referred to as BOOP - bronchiolitis obliterans organising pneumonia) has been reported in association with $\mathrm{CVID}^{[64]}$ (although therefore would not be considered "cryptogenic"). The diagnosis would be established at biopsy and organising pneumonia is also treated in the first instance with systemic corticosteroids.

Patients with CVID are at increased risk of lymphoma, typically B-cell NHL. MALT lymphoma may occur in the lung ${ }^{[65]}$ and should be considered in the differential diagnosis of GLILD. The management of intra-pulmonary lymphoma is out with the scope of this review.

\section{Processes of Care}


We advocate a shared care approach, ideally with joint respiratory-immunology clinics for patients with respiratory complications of PAD. This will facilitate access to a multi-professional team including radiologists, pathologists, and respiratory clinicians and respiratory physiotherapists in addition to the immunodeficiency team. Historically, there has been a delay both in patients with immunodeficiency seeing chest specialists (and chest specialists making the diagnosis of PAD in patients first presenting with respiratory pathology ${ }^{[22]}$.

\section{Key Research Questions}

It will be apparent from the above that key research questions remain regarding the optimal monitoring regime for lung disease in patients with $P A D$, and appropriate strategies for diagnosis and management. We propose the following key research priorities:

How can we best identify which PAD patients will retain healthy lungs, and which will develop bronchiectasis or GLILD?

What is the best prophylactic antibiotic management for patients with PAD and bronchiectasis?

What is the optimal management for patients with GLILD?

Is immunosuppression the only treatment for GLILD, and if so, which is the optimal immunosuppressive regimen?

How frequently should lung involvement be monitored by $\mathrm{CT}$ and lung function and in which patients is follow-up with MRI acceptable?

\section{Summary}

Respiratory manifestations of PAD are important because of the impact on morbidity and mortality. Bronchiectasis is the most common manifestation, with GLILD occurring in perhaps $15 \%$ of patients with CVID. The former should be managed according to standard guidelines, but evidence for effective diagnosis, monitoring and therapy of GLILD are lacking and remains an area of research priority.

\section{Acknowledgements}

We are grateful for the assistance of Sophie Pattison, Clinical Support Librarian, Medical Library, Royal Free Hospital for conducting the original literature search. 


\section{References}

1. Palmer DS, O'Toole J, Montreuil T et al. Screening of Canadian Blood Services donors for severe immunoglobulin A deficiency. Transfusion 2010;50:1524-1531

2. Eades-Perner AM, Gathmann B, Knerr V et al. The European internet-based patient and research database for primary immunodeficiencies: results 2004-06. Clin Exp Immunol 2007;147:306-312

3. Al-Saud BK, Al-Sum Z, Alassiri $\mathrm{H}$ et al. Clinical, immunological, and molecular characterization of hyper-IgM syndrome due to CD40 deficiency in eleven patients. Journal of Clinical Immunology 2013;33:1325-1335

4. ESID Registry - Working Definitions for Clinical Diagnosis of PID. Available at http://esid.org/Working-Parties/Registry/Diagnosis-criteria last accessed February 4th 2015

5. Winkelstein JA, Marino MC, Lederman HM et al. X-linked agammaglobulinemia: report on a United States registry of 201 patients. Medicine (Baltimore) 2006;85:193-202

6. Jorgensen GH, Gardulf A, Sigurdsson MI et al. Clinical symptoms in adults with selective IgA deficiency: a case-control study. Journal of Clinical Immunology 2013;33:742-747

7. Angulo I, Vadas O, Garcon F et al. Phosphoinositide 3-kinase gene mutation predisposes to respiratory infection and airway damage. Science 2013;342(6160):866-871

8. Jou ST, Chien YH, Yang YH et al. Identification of variations in the human phosphoinositide 3kinase p110delta gene in children with primary B-cell immunodeficiency of unknown aetiology. Int J Immunogenet. 2006 Oct;33(5):361-9.

9. Lucas $\mathrm{CL}$, Kuehn $\mathrm{HS}$, Zhao $\mathrm{F}$ et al. Dominant-activating germline mutations in the gene encoding the $\mathrm{PI}(3) \mathrm{K}$ catalytic subunit $\mathrm{p} 110 \delta$ result inT cell senescence and human immunod eficiency. Nat Immunol. 2014 Jan;15(1):88-97. doi: 10.1038/ni.2771.

10. Kracker S, Curtis J, Ibrahim MA et al. Occurrence of Bcell lymphomas in patients with activated phosphoinositide 3-kinase $\delta$ syndrome. J Allergy Clin Immunol. 2014 Jul;134(1):233-6. doi: 10.1016/j.jaci.2014.02.020.

11. Crank MC, Grossman JK, Moir S et al.

Mutations in PIK3CD can cause hyper IgM syndrome (HIGM) associated with increased cancersuscep tibility. J Clin Immunol. 2014 Apr;34(3):272-6. doi: 10.1007/s10875-014-0012-9.

12. Kuehn HS, Ouyang W, Lo B et al. Immune dysregulation in human subjects with heterozygous germline mutations in CTLA4. Science 2014;345:1623-1627

13. Schubert $D$, Bode $C$, Kenefeck $R$ et al. Autosomal dominant immune dysregulation syndrome in humans with CTLA4 mutations. Nat Med 2014;20:1410-1416 
14. Maglione PJ, Overbey JR, Radigan L, Bagiella E, Cunningham-Rundles C. Pulmonary radiologic findings in common variable immunodeficiency: clinical and immunological correlations. Annals of Allergy, Asthma, \& Immunology. 2014;113:452-459

15. Quinti I, Di Pietro C, Martini H et al. Health related quality of life in common variable immunodeficiency. Yonsei Medical Journal 2012;53:603-610

16. Hurst JR, Workman S, Garcha DS, Seneviratne SL, Haddock JA, Grimbacher B. Activity, severity and impact of respiratory disease in primary antibody deficiency syndromes. Journal of Clinical Immunology 2014;34:68-75

17. Resnick ES, Moshier El, Godbold JH, Cunningham-Rundles C. Morbidity and mortality in common variable immune deficiency over 4 decades. Blood 2012;119:1650-1657

18. Maarschalk-Ellerbroek LJ, De Jong PA, Van Montfrans JM et al. CT screening for pulmonary pathology in common variable immunodeficiency disorders and the correlation with clinical and immunological parameters. Journal of Clinical Immunology 2014;34:642-654

19. Aghamohammadi A, Allahverdi A, Abolhassani $\mathrm{H}$ et al. Comparison of pulmonary diseases in common variable immunodeficiency and X-linked agammaglobulinaemia. Respirology 2010;15:289295

20. Gregersen S, Aalokken TM, Mynarek $G$ et al. High resolution computed tomography and pulmonary function in common variable immunodeficiency. Respiratory Medicine 2009;103:873-880

21. Detkova D, De Gracia J, Lopes-Da-Silva S et al. Common variable immunodeficiency: association between memory B cells and lung diseases. Chest 2007;131:1883-1889

22. Thickett KM, Kumararatne DS, Banerjee AK, Dudley R, Stableforth DE. Common variable immune deficiency: respiratory manifestations, pulmonary function and high-resolution CT scan findings. Quarterly Journal of Medicine 2002;95:655-662

23. Gregersen S, Aalokken TM, Mynarek G et al. Development of pulmonary abnormalities in patients with common variable immunodeficiency: associations with clinical and immunologic factors. Ann Allergy Asthma Immunol 2010;104:503-10

24. Serra G, Milito C, Mitrevski M et al. Lung MRI as a possible alternative to CT scan for patients with primary immune deficiencies and increased radiosensitivity. Chest 2011;140:1581-1589

25. Chen Y, Stirling RG, Paul E, Hore-Lacy F, Thompson BR, Douglass JA. Longitudinal decline in lung function in patients with primary immunoglobulin deficiencies. Journal of Allergy and Clinical Immunology 2011;127:1414-1417

26. Rich Al, Le Jeune IR, Mcdermott L, Kinnear WJ. Serial lung function tests in primary immune deficiency. Clinical \& Experimental Immunology 2008;151:110-113

27. Menzin J, Sussman M, Munsell M, Zbrozek A. Economic impact of infections among patients with primary immunodeficiency disease receiving IVIG therapy. ClinicoEconomics and Outcomes Research 2014;6:297-302 
28. Busse PJ, Razvi S, Cunningham-Rundles C. Efficacy of intravenous immunoglobulin in the prevention of pneumonia in patients with common variable immunodeficiency. J Allergy Clin Immunol 2002;109:1001-100429. Lucas M, Lee M, Lortan J, Lopez-Granados E, Misbah S, Chapel H. Infection outcomes in patients with common variable immunodeficiency disorders: relationship to immunoglobulin therapy over 22 years. J Allergy Clin Immunol 2010;125:1354-1360

30. Orange JS, Grossman WJ, Navickis RJ, Wilkes MM. Impact of trough IgG on pneumonia incidence in primary immunodeficiency: a meta-analysis of clinical studies. Clin Immunol 2010;137:21-30

31. Quinti I, Soresina A, Guerra A et al. Effectiveness of immunoglobulin replacement therapy on clinical outcome in patients with primary antibody deficiencies: results from a multicenter prospective cohort study. Journal of Clinical Immunology 2011;31:315-322

32. Berger M. Incidence of infection is inversely related to steady-state (trough) serum IgG level in studies of subcutaneous IgG in PIDD. Journal of Clinical Immunology 2011;31:924-926

33. Kainulainen $\mathrm{L}$, Vuorinen $\mathrm{T}$, Rantokokko-Jalava $\mathrm{K}$ et al. Recurrent and persistent respiratory tract viral infections in patients with primary hypogammaglobulinemia. J Allergy Clin Immunol 2010;126:120-126

34. Kralickova P, Mala E, Vokurkova D et al. Cytomegalovirus disease in patients with common variable immunodeficiency: three case reports. International Archives of Allergy \& Immunology 2014;163:69-74

35. Pasteur MC, Bilton D, Hill AT. British Thoracic Society guideline for non-CF bronchiectasis. Thorax 2010,65(Suppl 1):i1-58

36. Hill AT, Routh C, Welham S. National BTS bronchiectasis audit 2012: Is the quality standard being adhered to in adult secondary care? Thorax 2014;69:292-2394

37. Gathmann B, Mahlaoui N, CEREDIH et al. Clinical picture and treatment of 2212 patients with common variable immunodeficiency. Journal of Allergy \& Clinical Immunology. 2014;134:116-126

38. Hampson FA, Chandra A, Screaton NJ et al. Respiratory disease in common variable immunodeficiency and other primary immunodeficiency disorders. Clinical Radiology 2012;67:587595

39. Serisier DJ, Martin ML, McGuckin MA et al. Effect of long-term, low-dose erythromycin on pulmonary exacerbations among patients with non-cystic fibrosis bronchiectasis: the BLESS randomized controlled trial. JAMA 2013;309:1260-1267

40. Wong $\mathrm{C}$, Jayaram $\mathrm{L}$, Karalus $\mathrm{N}$ et al. Azithromycin for prevention of exacerbations in non-cystic fibrosis bronchiectasis (EMBRACE): a randomised, double-blind, placebo-controlled trial. Lancet 2012;380:660-667

41. Altenburg J, de Graaff CS, Stienstra Y et al. Effect of azithromycin maintenance treatment on infectious exacerbations among patients with non-cystic fibrosis bronchiectasis: the BAT randomized controlled trial. JAMA 2013;309:1251-1259 
42. Fevang B, Mollnes TE, Holm AM et al. Common variable immunodeficiency and the complement system; low mannose-binding lectin levels are associated with bronchiectasis. Clinical \& Experimental Immunology 2005;142:576-84

43. Freiberger $T$, Grodecka L, Ravcukova B et al. Association of FcRn expression with lung abnormalities and IVIG catabolism in patients with common variable immunodeficiency. Clinical Immunology 2010;136:419-425

44. Kuruvilla M, De La Morena MT. Antibiotic prophylaxis in primary immune deficiency disorders. The Journal of Allergy \& Clinical Immunology in Practice 2013;1:573-582

45. Pereira AC, Kokron CM, Romagnolo BM et al. Analysis of the sputum and inflammatory alterations of the airways in patients with common variable immunodeficiency and bronchiectasis. Clinics (Sao Paulo, Brazil) 2009;64:1155-1160

46. Hurst JR. Microbial dysbiosis in bronchiectasis. Lancet Respir Med 2014;2:945-947

47. Kainulainen L, Nikoskelainen J, Vuorinen T, Tevola K, Liippo K, Ruuskanen O. Viruses and bacteria in bronchial samples from patients with primary hypogammaglobulinemia. American Journal of Respiratory and Critical Care Medicine 1999;159:1199-1204

48. Park JE, Beal I, Dilworth JP, Tormey V, Haddock J. The HRCT appearances of granulomatous pulmonary disease in common variable immune deficiency. European Journal of Radiology 2005;54:359-364

49. Maglione PJ, Ko HM, Beasley MB, Strauchen JA, Cunningham-Rundles C. Tertiary lymphoid neogenesis is a component of pulmonary lymphoid hyperplasia in patients with common variable immunodeficiency. Journal of Allergy \& Clinical Immunology 2014;133:535-542

50. Boursiquot JN, Gerard L, Malphettes M et al. Granulomatous disease in CVID: Retrospective analysis of clinical characteristics and treatment efficacy in a cohort of 59 patients. Journal of Clinical Immunology 2013;33:84-95

51. STILPAD Observational Study. Available at http://www.uniklinikfreiburg.de/cci/studien/stilpad.html last accessed February 4th 2015

52. Bouvry D, Mouthon L, Brillet PY et al. Granulomatosis-associated common variable immunodeficiency disorder: a case-control study versus sarcoidosis. European Respiratory Journal 2013;41:115-122

53. Kollert F, Venhoff N, Goldacker S et al. Bronchoalveolar lavage cytology resembles sarcoidosis in a subgroup of granulomatous CVID. European Respiratory Journal 2014;43:922-924

54. Naccache JM, Bouvry D, Valeyre D. Bronchoalveolar lavage cytology resembles sarcoidosis in a subgroup of granulomatous CVID. European Respiratory Journal 2014;43:924-925

55. Gregersen S, Holm AM, Fevang B et al. Lung disease, T-cells and inflammation in common variable immunodeficiency disorders. Scandinavian Journal of Clinical \& Laboratory Investigation 2013;73:514-522 
56. Kohler PF, Cook RD, Brown WR, Manguso RL. Common variable hypogammaglobulinemia with T-cell nodular lymphoid interstitial pneumonitis and B-cell nodular lymphoid hyperplasia: different lymphocyte populations with a similar response to prednisone therapy. J Allergy Clin Immunol 1982;70:299-305

57. Green H, Paul M, Vidal L, Leibovici L. Prophylaxis for Pneumocystis pneumonia (PCP) in non-HIV immunocompromised patients. Cochrane Database Syst Rev. 2007 Jul 18;(3).

58. Chase NM, Verbsky JW, Hintermeyer MK et al. Use of combination chemotherapy for treatment of granulomatous and lymphocytic interstitial lung disease (GLILD) in patients with common variable immunodeficiency (CVID). J Clin Immunol 2013;33:30-39

59. Davies CW, Juniper MC, Gray W, Gleeson FV, Chapel HM, Davies RJ. Lymphoid interstitial pneumonitis associated with common variable hypogammaglobulinaemia treated with cyclosporin A. Thorax 2000;55:88-90

60. Thatayatikom A, Thatayatikom S, White AJ. Infliximab treatment for severe granulomatous disease in common variable immunodeficiency: a case report and review of the literature. Annals of Allergy, Asthma, \& Immunology 2005;95:293-300

61. Wehr C, Gennery AR, Lindemans C et al. Multicenter experience in hematopoietic stem cell transplantation for serious complications of common variable immunodeficiency. J Allergy Clin Immunol 2015: in press [Epub ahead of print]

62. Bates CA, Ellison MC, Lynch DA, Cool CD, Brown KK, Routes JM. Granulomatous-lymphocytic lung disease shortens survival in common variable immunodeficiency. Journal of Allergy \& Clinical Immunology 2004;114:415-421

63. Burton $\mathrm{CM}$, Milman N, Andersen $\mathrm{CB}$, Marquart $\mathrm{H}$, Iversen $\mathrm{M}$. Common variable immune deficiency and lung transplantation. Scandinavian Journal of Infectious Diseases 2007;39:362-367

64. Kaufman J, Komorowski R. Bronchiolitis obliterans organizing pneumonia in common variable immunodeficiency syndrome. Chest 1991;2013:552-553

65. Reichenberger R, Wyser C, Gonon M, Cathomas G, Tamm M. Pulmonary mucosa-associated lymphoid tissue lymphoma in a patient with common variable immunodeficiency syndrome.

Respiration 2001;68:109-112 


\section{Appendix: Search Strategy}

Database: Ovid MEDLINE(R) Daily Update <November 19, 2014>, Ovid OLDMEDLINE(R) $<1946$ to 1965>, Ovid MEDLINE(R) In-Process \& Other Non-Indexed Citations and Ovid MEDLINE(R) $<1946$ to Present>

Search Strategy:

1 ((primary adj2 (antibody or immun*) adj2 deficienc*) or primary immunodeficienc*).ti,ab. (3325)

2 Immunologic Deficiency Syndromes/ (13104)

3 Common Variable Immunodeficiency/ (1592)

4 (common adj4 variable adj4 (immunodeficien* or immune deficien* or immuno-deficien*)).ti,ab. (1941)

5 CVID.ti,ab. (961)

6 Agammaglobulinemia/ (5776)

7 (hypogammaglobulin?emia* or agammaglobulin?emia*).ti,ab. (4462)

8 XLA.ti,ab. (460)

9 exp dysgammaglobulinemia/ (2961)

10 dysgammaglobulinemia?.ti,ab. (218)

11 Hyper IgM syndrome*.ti,ab. (394)

12 (HIGM? or XHIGM?).ti,ab. (150)

13 leukocyte-adhesion deficiency syndrome/ (405)

14 leukocyte-adhesion deficiency.ti,ab. (486)

15 phagocyte bactericidal dysfunction/ (605)

16 granulomatous disease, chronic/ (2821)

17 ((Bridges-Good or Quie or chronic granulomatous) adj (syndrome or disorder or disease)).ti,ab. (3081)

18 job syndrome/ (555)

19 ((hyper-IgE or hyperimmunoglobulinemia E or job or buckley) adj syndrome?).ti,ab. (508)

20 exp severe combined immunodeficiency/ (2681)

21 severe combined immunodeficienc*.ti,ab. (4271)

22 wiskott-aldrich syndrome/ (1353)

23 ((wiskott or Aldrich) adj syndrome).ti,ab. (1936)

24 T-Lymphocytopenia, Idiopathic CD4-Positive/ (228)

25 ((idiopathic or cd4?) adj2 (t-lymphocytop?enia? or lymphocytop?enia?)).ti,ab. (371)

26 or/1-25 (35842)

27 lung diseases/ (61262)

28 Respiratory Tract Infections/ (32172)

29 ((respiratory or lung) adj disease?).ti,ab. (56327)

30 exp Lung Diseases, Interstitial/ (48024)

31 exp Lung Diseases, Fungal/ (18385)

32 exp lung diseases, obstructive/ (176859)

33 exp Bronchiectasis/ (7757)

34 bronch?ect*.ti,ab. (7358)

35 exp pneumonia/ (77125)

36 pneumonia.ti,ab. (84950)

37 exp tuberculosis, pulmonary/ (68314)

38 tuberculosis.ti,ab. (152812)

39 Lung Abscess/ (4115)

40 ((lung or pulmonary) adj abscess*).ti,ab. (2621)

41 Pulmonary Fibrosis/ (16573)

42 Plasma Cell Granuloma, Pulmonary/ (249) 
43 ((pulmonary or lung*) adj2 ((inflammatory adj1 pseudotumo?r?) or (plasma cell adj1 granuloma?))).ti,ab. (111)

44 pulmonary fibros?s.ti,ab. (11613)

45 or/27-44 (621902)

4626 and 45 (3514)

47 limit 46 to animals (318)

48 limit 47 to humans (148)

4947 not $48(170)$

5046 not 49 (3344)

51 limit 50 to english language (2447)

52 limit 51 to "all child (0 to 18 years)" (1364)

53 limit 52 to "all adult (19 plus years)" (425)

5452 not 53 (939)

5551 not $54(1508)$

Database: Embase <1980 to 2014 Week 47>

Search Strategy:

1 ((respiratory or lung) adj disease?).ti,ab. (68861)

2 lung disease/ or autoimmune lung disease/ or exp bronchiolitis/ or chronic lung disease/ or chronic obstructive lung disease/ or exp interstitial lung disease/ or exp lung fibrosis/ or lung granuloma/ or exp lung infection/ or lung nodule/ or lung pseudotumor/ or lung sarcoidosis/ or multiple pulmonary nodules/ or exp pneumonia/ or small airway disease/ (476839)

3 exp bronchitis/ (46491)

4 exp bronchiectasis/ (13121)

5 bronch?ect*.ti,ab. (9042)

6 pneumonia.ti,ab. (103966)

7 tuberculosis.ti,ab. (151935)

8 ((lung or pulmonary) adj abscess*).ti,ab. (2738)

9 ((pulmonary or lung*) adj2 ((inflammatory adj1 pseudotumo?r?) or (plasma cell adj1 granuloma?))).ti,ab. (131)

10 pulmonary fibros?s.ti,ab. (14275)

11 or/1-10 (646435)

12 ((primary adj2 (antibody or immun*) adj2 deficienc*) or primary immunodeficienc*).ti,ab. (4834)

13 (common adj4 variable adj4 (immunodeficien* or immune deficien* or immunodeficien*)).ti,ab. (2639)

14 CVID.ti,ab. (1699)

15 exp agammaglobulinemia/ or exp dysgammaglobulinemia/ (9569)

16 (hypogammaglobulin?emia* or agammaglobulin?emia*).ti,ab. (5130)

17 XLA.ti,ab. (606)

18 dysgammaglobulinemia?.ti,ab. (204)

19 Hyper IgM syndrome*.ti,ab. (487)

20 (HIGM? or XHIGM?).ti,ab. (200)

21 combined immunodeficiency/ or exp severe combined immunodeficiency/ or wiskott aldrich syndrome/ (9293)

22 exp leukocyte adhesion deficiency/ (677)

23 leukocyte-adhesion deficiency.ti,ab. (571)

24 phagocyte dysfunction/ or chronic granulomatous disease/ or granulocytopenia/ or hyper ige syndrome/ (9273) 
25 ((Bridges-Good or Quie or chronic granulomatous) adj (syndrome or disorder or disease)).ti,ab. (3431)

26 ((hyper-IgE or hyperimmunoglobulinemia E or job or buckley) adj syndrome?).ti,ab. (744)

27 severe combined immunodeficienc*.ti,ab. (4594)

28 ((wiskott or Aldrich) adj syndrome).ti,ab. (2103)

29 ((idiopathic or cd4?) adj2 (t-lymphocytop?enia? or lymphocytop?enia?)).ti,ab. (451)

30 or/12-29 (37024)

3111 and $30(4726)$

32 limit 31 to english language (4186)

33 limit 32 to (embryo < first trimester $>$ or infant <to one year $>$ or child < unspecified age $>$ or preschool child $<1$ to 6 years $>$ or school child $<7$ to 12 years $>$ or adolescent $<13$ to 17 years $>$ ) (1258)

34 limit 33 to (adult $<18$ to 64 years $>$ or aged $<65+$ years $>$ ) ( 371 )

3533 not $34(887)$

$36 \quad 32$ not 35 (3299)

37 limit 36 to animals (165)

3836 not 37 (3134)

39 limit 38 to conference abstract (774)

4038 not $39(2360)$ 